\title{
Stochastic approach to the Navier-Stokes equation
}

\author{
2021/04/29 First Edition \\ Takuya Yabu(takuya.yabu@live.jp)
}

\begin{abstract}
Take a stochastic approach to the Navier-Stokes equation. The pressure and flow velocity are used as probabilities, the $x y z$ coordinates are replaced with molar concentrations, and the Navier-Stokes equation is transformed into the Fokker-Planck equation using Ito's lemma(formula). This made it possible to obtain the probability of turbulence from the Navier-Stokes equation. The molar concentration in the micro space can be obtained by separately solving the diffusion equation. Using these results, the probability of turbulence and the quantities such as fluid pressure and flow velocity can be analytically obtained.
\end{abstract}

\section{Introduction}

Attempts have been made to find the state quantity of a fluid by solving the Navier-Stokes equation. Mohan considered the three-dimensional Navier-Stokes equations on the whole space with a hereditary viscous term which depends on the past history (Mohan, 2020). On the stochastic tamed Navier-Stokes equations, the existence and the uniqueness of solutions and the existence of an invariant measure were improved (Brzeźniak \& Dhariwal, 2020). Colombo and Haffter considered solutions of the Navier-Stokes equation with fractional dissipation (Colombo \& Haffter, 2021). Weak solutions of the 3D Navier-Stokes equations were not unique in the class of weak solutions with finite kinetic energy (Buckmaster \& Vicol, 2019). The intrinsic relation between Navier-Stokes equations and dynamical density functional theory (DDFT) was revealed (Qiao, et al., 2021). Similar to solving analytically, it is also actively found by simulation. Lemoine \& Münch improved a least-squares method in order to solve implicit time schemes associated to the 2D and 3D Navier-Stokes system (Lemoine \& Münch , 2021). Chu, et al evaluated the fractional-order multi-dimensional NavierStokes equations using a variational iteration transform technique (Chu, Ali Shah, Agarwal, \& Chung, 2021). Modifying the classic multi-phase-field elastic bending energy model to establish a new volume-conserved mode, and developing a new decoupling method for the flow-coupled model, and combined it with other proven effective numerical methods, to form an efficient numerical scheme (Yang, 2021). Reuther and Voigt considered a numerical approach for the incompressible surface Navier-Stokes equation on surfaces (Reuther \& Voigt, 2018). The modification of 
Adomian decomposition method to solve fractional-order multi-dimensional NavierStokes equation were proposed (Shaha, Khana, Baleanubc, Kumamde, \& Arifa, 2020). Abbaszadeh and Dehghan proposed a meshless weak form to simulate the Oldroyd equation (Abbaszadeh \& Dehghan, 2020). However, as far as the author knows, there is no stochastic approach to determine the probability of turbulence. Take a stochastic approach to the Navier-Stokes equation. The pressure and flow velocity are replacing with probabilities, the $x y z$ coordinates are replaced with molar concentrations, and the Navier-Stokes equation is transformed into the Fokker-Planck equation using Ito's lemma(formula). As a result, the probability of turbulence can be obtained from the Navier-Stokes equation, and the pressure and flow velocity can be obtained from the obtained probability. In this paper, I will discuss specific methods in the following.

2. Transformation of the Navier-Stokes equation

The Navier-Stokes equation is given below. Here, $\frac{D}{D t}$ is the Lagrangian derivative.

$$
\frac{D \vec{v}}{D t}=\frac{\partial \vec{v}}{\partial t}+(\vec{v} \cdot \nabla) \vec{v}=F-\frac{1}{\rho} \nabla p+\frac{1}{3} \nu \nabla(\nabla \cdot \vec{v})+\nu \nabla^{2} \vec{v}
$$

Divide both sides by $p_{\infty},\left|\vec{v}_{\infty}\right|$, which is a large value such that $p$ or $\vec{v}$ is the maximum value of 1 . Also, set $F=0$.

$$
\begin{aligned}
\frac{1}{p_{\infty}} \frac{\partial}{\partial t}\left(\frac{\vec{v}}{\left|\vec{v}_{\infty}\right|}\right)+ & \frac{1}{p_{\infty}}(\vec{v} \cdot \nabla) \frac{\vec{v}}{\left|\vec{v}_{\infty}\right|} \\
& =-\frac{1}{\rho\left|\vec{v}_{\infty}\right|} \nabla \frac{p}{p_{\infty}}+\frac{1}{3} \frac{v}{p_{\infty}} \nabla\left(\nabla \cdot \frac{\vec{v}}{\left|\vec{v}_{\infty}\right|}\right)+\frac{v}{p_{\infty}} \nabla^{2} \frac{\vec{v}}{\left|\vec{v}_{\infty}\right|}
\end{aligned}
$$

Further formula transformation is performed.

$$
\begin{aligned}
\frac{1}{p_{\infty}} \frac{\partial}{\partial t}\left(1-\frac{\vec{v}}{\left|\vec{v}_{\infty}\right|}\right) & +\frac{1}{p_{\infty}}(\vec{v} \cdot \nabla)\left(1-\frac{\vec{v}}{\left|\vec{v}_{\infty}\right|}\right) \\
= & -\frac{1}{\rho\left|\vec{v}_{\infty}\right|} \nabla\left(1-\frac{p}{p_{\infty}}\right)+\frac{1}{3} \frac{v}{p_{\infty}} \nabla\left(\nabla \cdot\left(1-\frac{\vec{v}}{\left|\vec{v}_{\infty}\right|}\right)\right) \\
& +\frac{v}{p_{\infty}} \nabla^{2}\left(1-\frac{\vec{v}}{\left|\vec{v}_{\infty}\right|}\right)
\end{aligned}
$$

Now consider the following equation. $p_{r}$ and $\overrightarrow{p_{r}}$ mean the probability of turbulence.

$$
p_{r}=1-\frac{p}{p_{\infty}}, \overrightarrow{p_{r}}=1-\frac{\vec{v}}{\left|\vec{v}_{\infty}\right|}
$$

Therefore, the following equation is obtained.

$$
\frac{1}{p_{\infty}} \frac{\partial \overrightarrow{p_{r}}}{\partial t}+\frac{1}{p_{\infty}}(\vec{v} \cdot \nabla) \overrightarrow{p_{r}}=-\frac{1}{\rho\left|\vec{v}_{\infty}\right|} \nabla p_{r}+\frac{1}{3} \frac{v}{p_{\infty}} \nabla\left(\nabla \cdot \overrightarrow{p_{r}}\right)+\frac{v}{p_{\infty}} \nabla^{2} \overrightarrow{p_{r}}
$$

Now, think about $\nabla \cdot \overrightarrow{p_{r}}$ The probability of turbulence does not increase or decrease in 
a micro space. Therefore, $\nabla \cdot \overrightarrow{p_{r}}=0$ Therefore, the following equation is obtained.

$$
\frac{1}{p_{\infty}} \frac{\partial \overrightarrow{p_{r}}}{\partial t}+\frac{1}{p_{\infty}}(\vec{v} \cdot \nabla) \overrightarrow{p_{r}}=-\frac{1}{\rho\left|\vec{v}_{\infty}\right|} \nabla p_{r}+\frac{v}{p_{\infty}} \nabla^{2} \overrightarrow{p_{r}}
$$

Here, considering the $x$ coordinate of the $x y z$ coordinates, the following equation can be obtained.

$$
\begin{aligned}
\frac{1}{p_{\infty}} \frac{\partial p_{r_{x}}}{\partial t}+\frac{1}{p_{\infty}}\left(v_{x} \frac{\partial p_{r_{x}}}{\partial x}+v_{y} \frac{\partial p_{r_{x}}}{\partial y}+v_{z} \frac{\partial p_{r_{x}}}{\partial z}\right) \\
=-\frac{1}{\rho\left|\vec{v}_{\infty}\right|} \frac{\partial p_{r}}{\partial x}+\frac{v}{p_{\infty}}\left(\frac{\partial^{2} p_{r_{x}}}{\partial x^{2}}+\frac{\partial^{2} p_{r_{x}}}{\partial y^{2}}+\frac{\partial^{2} p_{r_{x}}}{\partial z^{2}}\right)
\end{aligned}
$$

Since the $x$ coordinate is not a random variable, it is converted to a random variable $X$ by the following formula. Here, $W_{T}$ is a Brownian motion for time $T$.

$$
d X=\Delta x \cdot d W_{T}
$$

Therefore, the following equation can be obtained in a micro space.

$$
\frac{\partial X}{\partial x}=d W_{T}
$$

Similarly, the following equation is obtained. Here, let $Y$ be a random variable of $y$ and $Z$ be a random variable of $z$.

$$
\begin{aligned}
& \frac{\partial Y}{\partial y}=d W_{T} \\
& \frac{\partial Z}{\partial z}=d W_{T}
\end{aligned}
$$

Furthermore, consider the time $t$. The following equation is obtained using the scalar $\Delta T$ between the time $T$ and the random variable.

$$
d T=\Delta T \cdot d t
$$

Therefore, considering a very small amount of time, the following equation can be obtained.

$$
\frac{\partial T}{\partial t}=d T
$$

Therefore, from equations (9), (10), (11), and (13), equation ( 7) can be transformed as follows.

$$
\begin{aligned}
\frac{1}{p_{\infty}} \frac{\partial p_{r_{x}}}{\partial T} d T+\frac{1}{p_{\infty}} & \left(v_{x} \frac{\partial p_{r_{x}}}{\partial X}+v_{y} \frac{\partial p_{r_{x}}}{\partial Y}+v_{z} \frac{\partial p_{r_{x}}}{\partial Z}\right) d W_{T} \\
& =-\frac{1}{\rho\left|\vec{v}_{\infty}\right|} \frac{\partial p_{r}}{\partial X} d W_{T} \\
& +\frac{v}{p_{\infty}}\left(\frac{\partial^{2} p_{r_{x}}}{\partial X^{2}}+\frac{\partial^{2} p_{r_{x}}}{\partial Y^{2}}+\frac{\partial^{2} p_{r_{x}}}{\partial Z^{2}}\right)\left(d W_{T}\right)^{2}
\end{aligned}
$$


Here, Ito's lemma(formula) is used (Oksendal, 2003). $\left(d W_{T}\right)^{2}=d T$.

$$
\begin{aligned}
\frac{1}{p_{\infty}} \frac{\partial p_{r_{x}}}{\partial T} d T-\frac{v}{p_{\infty}} & \left(\frac{\partial^{2} p_{r_{x}}}{\partial X^{2}}+\frac{\partial^{2} p_{r_{x}}}{\partial Y^{2}}+\frac{\partial^{2} p_{r_{x}}}{\partial Z^{2}}\right) d T \\
= & -\frac{1}{\rho\left|\vec{v}_{\infty}\right|} \frac{\partial p_{r}}{\partial X} d W_{T} \\
& +\frac{1}{p_{\infty}}\left(v_{x} \frac{\partial p_{r_{x}}}{\partial X}+v_{y} \frac{\partial p_{r_{x}}}{\partial Y}+v_{z} \frac{\partial p_{r_{x}}}{\partial Z}\right) d W_{T}
\end{aligned}
$$

Integrate both sides from 0 to $\infty$.

$$
\begin{aligned}
\int_{0}^{\infty} \frac{1}{p_{\infty}} \frac{\partial p_{r_{x}}}{\partial T} d T & -\int_{0}^{\infty} \frac{v}{p_{\infty}}\left(\frac{\partial^{2} p_{r_{x}}}{\partial X^{2}}+\frac{\partial^{2} p_{r_{x}}}{\partial Y^{2}}+\frac{\partial^{2} p_{r_{x}}}{\partial Z^{2}}\right) d T \\
& =-\int_{0}^{\infty} \frac{1}{\rho\left|\vec{v}_{\infty}\right|} \frac{\partial p_{r}}{\partial X} d W_{T} \\
& +\int_{0}^{\infty} \frac{1}{p_{\infty}}\left(v_{x} \frac{\partial p_{r_{x}}}{\partial X}+v_{y} \frac{\partial p_{r_{x}}}{\partial Y}+v_{z} \frac{\partial p_{r_{x}}}{\partial Z}\right) d W_{T}
\end{aligned}
$$

Furthermore, $h(X, T)$ is multiplied on both sides to perform integration by parts.

$$
\begin{aligned}
-\int_{0}^{\infty} \frac{1}{p_{\infty}} p_{r_{x}} \frac{\partial h}{\partial T} & d T-\int_{0}^{\infty} \frac{v}{p_{\infty}} p_{r_{x}}\left(\frac{\partial^{2} h}{\partial X^{2}}+\frac{\partial^{2} h}{\partial Y^{2}}+\frac{\partial^{2} h}{\partial Z^{2}}\right) d T \\
= & \int_{0}^{\infty} \frac{1}{\rho\left|\vec{v}_{\infty}\right|} p_{r} \frac{\partial h}{\partial X} d W_{T} \\
& -\int_{0}^{\infty} \frac{1}{p_{\infty}} p_{r_{x}}\left(v_{x} \frac{\partial h}{\partial X}+v_{y} \frac{\partial h}{\partial Y}+v_{z} \frac{\partial h}{\partial Z}\right) d W_{T}
\end{aligned}
$$

Take the expected value for $X$ on both sides. Since the right side is 0 , the following equation is obtained.

$$
\int_{-\infty}^{\infty} d X \int_{0}^{\infty} d T p_{r_{x}}\left(-\frac{1}{p_{\infty}} \frac{\partial h}{\partial T}-\frac{v}{p_{\infty}}\left(\frac{\partial^{2} h}{\partial X^{2}}+\frac{\partial^{2} h}{\partial Y^{2}}+\frac{\partial^{2} h}{\partial Z^{2}}\right)\right)=0
$$

Perform integration by parts again.

$$
\int_{-\infty}^{\infty} d X \int_{0}^{\infty} d T h(X, T)\left(\frac{1}{p_{\infty}} \frac{\partial p_{r_{x}}}{\partial T}-\frac{v}{p_{\infty}}\left(\frac{\partial^{2} p_{r_{x}}}{\partial X^{2}}+\frac{\partial^{2} p_{r_{x}}}{\partial Y^{2}}+\frac{\partial^{2} p_{r_{x}}}{\partial Z^{2}}\right)\right)=0
$$

In order for equation (19) to hold for almost any $h(X, T)$, the integrand must be zero. Therefore, the following equation is obtained.

$$
\frac{1}{p_{\infty}} \frac{\partial p_{r_{x}}}{\partial T}=\frac{v}{p_{\infty}}\left(\frac{\partial^{2} p_{r_{x}}}{\partial X^{2}}+\frac{\partial^{2} p_{r_{x}}}{\partial Y^{2}}+\frac{\partial^{2} p_{r_{x}}}{\partial Z^{2}}\right)
$$

Therefore, the following equation holds.

$$
\frac{\partial p_{r_{x}}}{\partial T}=v\left(\frac{\partial^{2} p_{r_{x}}}{\partial X^{2}}+\frac{\partial^{2} p_{r_{x}}}{\partial Y^{2}}+\frac{\partial^{2} p_{r_{x}}}{\partial Z^{2}}\right)
$$

The same applies to the $y$ and $z$ coordinates. 


$$
\begin{aligned}
& \frac{\partial p_{y}}{\partial T}=v\left(\frac{\partial^{2} p_{r_{y}}}{\partial X^{2}}+\frac{\partial^{2} p_{r_{y}}}{\partial Y^{2}}+\frac{\partial^{2} p_{r_{y}}}{\partial Z^{2}}\right) \\
& \frac{\partial p_{r_{z}}}{\partial T}=v\left(\frac{\partial^{2} p_{r_{z}}}{\partial X^{2}}+\frac{\partial^{2} p_{z}}{\partial Y^{2}}+\frac{\partial^{2} p_{r_{z}}}{\partial Z^{2}}\right)
\end{aligned}
$$

Therefore, since these equations ( 21), (22), and (23) are the same equations, they can be unified by the following equations.

$$
\frac{\partial p_{r}}{\partial T}=v\left(\frac{\partial^{2} p_{r}}{\partial X^{2}}+\frac{\partial^{2} p_{r}}{\partial Y^{2}}+\frac{\partial^{2} p_{r}}{\partial Z^{2}}\right)
$$

Now, suppose that $\alpha$ mol increases or decreases from the $X$-axis, $\beta$ mol from the $Y$-axis, and $\gamma$ mol from the $Z$-axis in a minute space. From this, assuming that the number of moles of particles in a micro space is $n$, the following equation holds.

$$
\frac{\partial n}{\partial X}=\alpha, \frac{\partial n}{\partial Y}=\beta, \frac{\partial n}{\partial Z}=\gamma
$$

Therefore, from equations ( 24) and ( 25), the following equation holds. However, let $D=2 v\left(\alpha^{2}+\beta^{2}+\gamma^{2}\right)$. This is the Fokker-Planck equation obtained from the Navier-Stokes equation.

$$
\frac{\partial p_{r}}{\partial T}=\frac{1}{2} D \frac{\partial^{2} p_{r}}{\partial n^{2}}
$$

From equation ( 26 ), the following equation is obtained. However, $n \geq 0$. Note that $D(X, Y, Z)=2 v\left(\alpha(X)^{2}+\beta(Y)^{2}+\gamma(Z)^{2}\right)$ may be used here.

$$
p_{r}(n, T)=\frac{2}{\sqrt{2 \pi D T}} e^{-\frac{n^{2}}{2 D T}}
$$

From equation (27), the probability of turbulence approaches 0 over time. This means that the diffusion of the fluid into the region increases the probability of laminar flow rather than turbulence. Further, it can be seen that the larger the molar concentration $\mathrm{n}$ in the micro space, the smaller the movement of the particles in the micro space, so that the probability of turbulence is small and laminar flow is likely to occur.

The molar concentration $n$ in the region can be obtained from the diffusion equation. If the molar concentration $\mathrm{n}$ is known, the probability of turbulence in a minute space can be obtained from equation ( 27). Also, given the boundary conditions, $p_{\infty}$, $v_{x_{\infty}}, v_{y_{\infty}}, v_{z_{\infty}}$. can be obtained from equation. (4) using the probability at the boundary. Therefore, the pressure and the flow velocity in the region can be obtained.

\section{Conclusion}

I took a stochastic approach to the Navier-Stokes equation. I replaced pressure and flow velocity with probability of turbulence, $x y z$ coordinates with molarity, and 
transformed the Navier-Stokes equation into the Fokker-Planck equation using Ito's lemma(formula). This made it possible to obtain the probability of turbulence from the Navier-Stokes equation. The molar concentration in the micro space can be obtained by separately solving the diffusion equation. Using these results, it has become possible to analytically determine the probability of turbulence and the quantities such as fluid pressure and flow velocity.

\section{References}

Abbaszadeh, M., \& Dehghan, M. (2020). Investigation of the Oldroyd model as a generalized incompressible Navier-Stokes equation via the interpolating stabilized element free Galerkin technique. Applied Numerical Mathematics, Volume 150.

Brzeźniak, Z., \& Dhariwal, G. (2020). Stochastic Tamed Navier-Stokes Equations on R3 : The Existence and the Uniqueness of Solutions and the. Journal of Mathematical Fluid Mechanics, Volume 22, No. 23.

Buckmaster, T., \& Vicol, V. (2019). Nonuniqueness of weak solutions to the Navier-Stokes equation. Annals of Mathematics, Volume 189, No. 1.

Chu, Y.-M., Ali Shah, N., Agarwal, P., \& Chung, J. (2021). Analysis of fractional multi-dimensional Navier-Stokes equation. Advances in Difference Equations, Article number: 91(2021).

Colombo, M., \& Haffter, S. (2021). Global regularity for the hyperdissipative Navier-Stokes equation below the critical order. Journal of Differential Equations, Volume 275.

Lemoine, J., \& Münch , A. (2021). Resolution of the implicit Euler scheme for the Navier-Stokes equation through a least-squares method. Numerische Mathematik, Volume 147.

Mohan, M. (2020). An extension of the Beale-Kato-Majda criterion for the $3 D$ Navier-Stokes equation with hereditary viscosity. Pure and Applied Functional Analysis, Volume 5, No. 2.

Oksendal, B. (2003). Stochastic Differential Equations: An Introduction with Applications. Springer.

Qiao, C., Zhao, T., Yu, X., Qing, L., Bao, B., Zhao, S., \& Liu, H. (2021). On the relation between dynamical density functional theory and Navier-Stokes equation. Chemical Engineering Science, Volume 230.

Reuther, S., \& Voigt, A. (2018). Solving the incompressible surface Navier-Stokes equation by surface finite elements. Physics of Fluids, 30. 
Shaha, R., Khana, H., Baleanubc, D., Kumamde, P., \& Arifa, M. (2020). The analytical investigation of time-fractional multi-dimensional Navier-Stokes equation. Alexandria Engineering Journal, Volume 59, Issue 5.

Yang, X. (2021). Numerical approximations of the Navier-Stokes equation coupled with volume-conserved multi-phase-field vesicles system: Fully-decoupled, linear, unconditionally energy stable and second-order time-accurate numerical scheme. Computer Methods in Applied Mechanics and Engineering, Volume 375. 